\title{
New approaches for integrated and cost-effective malaria vector control
} Peter Dambach ${ }^{*}$

'Institute of Public Health, University of Heidelberg, Germany

Article Info

\section{Article Notes}

Received: January 15, 2018

Accepted: February 12, 2018

\section{*Correspondence:}

Dr. Peter Dambach, Institute of Public Health, University of Heidelberg, Germany, E-mail: peter.dambach@web.de

C 2018 Dambach P. This article is distributed under the terms of the Creative Commons Attribution 4.0 International License.

\section{Keywords}

Biological larviciding

Insect growth regulators

Bacillus thuringiensis israelensis

Sub-Saharan Africa

Cost effectiveness

Multi disease vector control

Malaria

Dengue

\section{ABSTRACT}

In most parts of sub-Saharan Africa, malaria is still the most important vector borne disease, severely affecting people's lives and causing economic loss. Malaria vector control to date almost exclusively relies on long lasting insecticide treated nets (LLINs) and indoor residual spraying (IRS), while other approaches such as larviciding are less implemented. Rising resistances against commonly used insecticides and changes in vector behavior and genetics slow down further reductions in malaria transmission. There is an urgent need for the implementation of additional approaches to appropriately react to vector adaptations. One promising option is the targeting of vector larvae with biological larvicides. During a three-year field trial (EMIRA - Ecologic Malaria Reduction for Africa), evidence was generated on the feasibility, effectiveness, acceptability, and cost of biological larviciding in North-Western Burkina Faso. Here, possible ways on how to further increase cost effectiveness and community support for future programs are presented. Reducing the need for frequent retreatment of vector larvae habitats is a major cost saver for material and workforce. Additional expenditure reductions could be achieved through targeting multiple disease vectors, which in some cases share vector breeding and resting sites. Due to limited overlap in mosquito breeding preference, major cost savings are expected to originate in infrastructural synergy effects. The development of new approaches to further cut down program costs could be a powerful contributor to promote vector larvae control and techniques that target several diseases at once.

\section{Current challenges in malaria vector control}

Malaria remains to be the most important vector borne disease in sub-Saharan Africa (SSA) with an estimated 194 million yearly cases and 346,000 deaths in this region ${ }^{1}$. Today's efforts in controlling malaria vector mosquitoes predominantly rely on the distribution and use of long lasting insecticide treated nets (LLINs) and to a smaller extent on indoor residual spraying (IRS) with insecticides. For Africa, the attributable effect of insecticide treated nets and IRS on achieved malaria prevalence reductions between 2010 and 2015 were estimated at $68 \%$ and $13 \%$ respectively ${ }^{2}$. The reduction of vector populations at their larval stages on the other hand has received less attention after successful undertakings in the early $20^{\text {th }}$ century and since then has been implemented only by few African national malaria control programs covering limited areas of the country. Although the targeting of adult vector populations is generally seen as more effective compared to larval control $^{3}$, there is consent that vector control should be integrated, comprising adult and larval stages ${ }^{4}$. After decades of optimism to further curb malaria, which was largely nurtured by the success of 
bed net campaigns, today vector control faces increased headwind once again. Although new research indicates that resistances of mosquitoes against insecticides might not compromise bed net and spraying campaigns as much as often reported ${ }^{5-7}$, those developments together with changes in vector behavior and alterations of vector species compositions may adversely affect current control efforts $^{7-11}$. Improved biological larval control methods are a promising approach to fill in at the weak spots of adult control interventions that are currently in place. Biological larvicides such as Bacillus thuringiensis israelensis (Bti) are environmentally safe, target species specific in their mode of action, and show no resistances ${ }^{12}$. Within the last decade there has been a large body of evidence generated, showing that Bti based larviciding can bring down malaria vector populations by order of magnitude and significantly reduce malaria prevalence ${ }^{13-16}$. Recommendations from the WHO currently limit the use of larviciding to urban environments due to the high population density, which provides lower per capita intervention costs compared to less densely populated areas ${ }^{4}$. Despite those very focal recommendations, there are several examples of successful implementation in rural areas of sub-Saharan Africa, while environments with heavy and year round flooding seem inappropriate for larviciding ${ }^{17}$. Furthermore, Bti based larviciding can be provided at low cost, comparable to, or lower than those of LLINs ${ }^{18-20}$. A common misconception is that reinforcing efforts in larval control inevitably would pull away resources from adult vector control programs. In fact, the contrary should be performed in practice, combining anti-adult and anti-larvae strategies. Mathematical models indeed corroborate observations of disproportionally high mosquito reductions of integrated vector control ${ }^{21}$. Whether larviciding will receive again the propagation and role in malaria control it once had, depends not least on its implementation costs. Here some observations from a large scale larviciding trial are used to explore new ways of reducing implementation costs for such programs and hence facilitate their uptake into national malaria control programs and practical implementation.

Improving cost effectiveness - Implications of the EMIRA project

Within a three-year research project in rural Burkina Faso (EMIRA - Ecologic Malaria Reduction for Africa), the feasibility of $B t i$ based larviciding was investigated in a resource restraint setting in North-Western Burkina Faso ${ }^{22}$. This intervention achieved a reduction in female vector mosquitoes of more than $75 \%$ compared to untreated control areas at yearly per capita costs of US $\$ 1.10^{18,23}$. Besides entomological and epidemiological impact, costeffectiveness is key to make larviciding competitive with other approaches and facilitate uptake into national and regional control programs. Following this line of argumentation, possible cost savings through the selective treatment of larval breeding sites were investigated. Remote sensing derived risk maps were used to identify breeding sites with the highest larval productivity ${ }^{24-26}$. Cost savings turned out to be disproportionally higher than the increase in mosquito numbers due to the omission of treating breeding sites. Ideally, cost saving approaches should not compete with effectiveness. In our study area, it turned out to be difficult to rely on a selective treatment approach, since virtually all stagnant water bodies harbored Anopheles larvae, even if at low densities. During project impact evaluation and the analysis of implementation costs, three major areas were identified that could be modified for increased efficacy and cost-efficiency in future projects.

Firstly, the short residual effect of Bti under tropical climatic conditions calls for frequent retreatment of breeding sites, roughly every 8 to 10 days $^{27,28}$. Reducing the frequency of treatment intervals would significantly reduce the cost for manpower, transportation, and depending on the used product, the cost of larvicides. Therefore, novel formulations of $B t i$ that disperse the active ingredients over a longer time seem to be an attractive option. However, a limiting factor for the deployment of time release tablets or floating granules is the nature of typical Anopheles breeding sites in many parts of the Sahel. A pilot study in Burkina Faso (Dambach, unpublished data) showed wind drift of time release granules (VectoMax FG, Valent BioSciences) from the windward to the leeward side of the breeding site several meters away, within minutes. This resulted in non-lethal dosages in the windward half of breeding sites, while in the leeward parts, mosquito larvae died after few hours. A novel and promising approach are insect growth regulators that inhibit molting of target organisms such as mosquitoes. Recent research showed that via using those substances, the retreatment interval of spraying breeding sites could be extended from 8 to 10 days to 4 to 6 weeks depending on breeding site ecology ${ }^{29,30}$. Despite relatively low costs for manpower in community supported programs, extended treatment intervals could help to drastically cut on routine implementation costs. Equally helpful in minimizing infrastructure cost is the ability of these formulations to regain efficacy during flooding after having fallen dry. This is particularly interesting in settings that feature smaller breeding sites that can fall dry during few days with no precipitation and refill afterward. Although growth regulators such as pyriproxyfen and s-metoprene are expensive, they could prove helpful in drastically reducing treatment $\operatorname{cost}^{30}$. Furthermore, the use of growth regulators is not limited to the administration to breeding sites but can be extended to adult vector control in integrated approaches. Insect growth regulators are available for different modes of application, ranging from time release granules to powders and have recently been used at large scale to curb the zika epidemic in Brazil ${ }^{31}$. 
Recent research unveiled the possibility of so called "auto dissemination" of growth regulators during which mosquitoes carry small amounts of the substance to tiny, normally non accessible breeding sites ${ }^{32-34}$.

Second, the EMIRA project targeted primarily malaria mosquitoes, breeding in puddles and ponds within and around villages. Breeding sites in private compounds such as pit latrines and small breeding containers were not treated. This resulted in a discrepancy in vector reductions depending on species. While malaria transmitting Anopheles mosquitoes were reduced by more than $75 \%$, Culex and Aedes mosquitoes were impacted much less, with only $34 \%$ achieved reduction ${ }^{23}$. Although mosquito species that favor these types of breeding sites, predominantly Culex and Aedes, do not transmit malaria, they are a major nuisance. In-depth interviews (II), focus group discussions (FGD), and guided questionnaires were used to gather data on the perceived success of anti-malarial larviciding programs. A total of 634 questionnaire interviews and 12 FGDs and IIs each which were performed in treatment and untreated control arms, indicated, that those programs could benefit from equally treating Culex and Aedes larval sources, since community members were not able to discriminate between different mosquito species and attribute a corresponding risk ${ }^{35}$. The success of larviciding programs heavily depends on community acceptance and their willingness of getting involved ${ }^{36-38}$. Support, financially, through labor, or by granting access to private compounds, is more likely to be offered by the communities if people can witness an immediate relief in mosquito nuisance. Therefore, future undertakings should include targeting of non-malaria mosquitoes. Although no direct cost savings are likely to be expected for malaria control, a more comprehensive view on the disease situation in many sub-Saharan regions unveils possible cost savings indeed.

Thirdly, many areas of sub-Saharan Africa increasingly experience severe health and economic damage through other vector borne diseases such as dengue fever and chikungunya ${ }^{39,40}$. In contrast to Asia and South America, where non-malaria mosquito control is widely in place and financially supported, it is virtually absent in SSA. Addressing the increasing momentum of dengue and other diseases in the wake of climate change in an often resource restraint African setting, it would be beneficial to bundle up efforts in a way that the same infrastructure can be used for targeting multiple vector borne diseases. Although Aedes, Anopheles and Culex mosquitoes were found to have their unique breeding habitats within the study region, there was strong overlap between Culex and Anopheles mosquitoes in non-polluted puddles and ponds, which make up the highest share of water bodies in this area. Cost reductions are hence to be expected predominantly from infrastructural synergy effects, using already implemented program structure to additionally treat further vector habitats.

Control programs for virtually all tropical mosquito borne diseases depend on tightly organized field interventions. Despite the existing variety of those diseases, the most effective interventions are condensing down to a few approaches, amongst which are indoor residual spraying, insecticide treated nets and the treatment of larval habitats $^{41}$. For many settings, large parts of the program infrastructure are identical, including personnel for project management, field applications, community mobilization and transport costs. However, arboviral diseases such as chikungunya and dengue are subject to different transmission dynamics, which are often characterized by outbreaks following longer periods of lower transmission. Although dengue and chikungunya vector control have some overlap with that for malaria, their complexity ideally calls for the additional implementation and financing of continuous surveillance or early warning systems. Those systems are able to capture spatial and temporal variations in disease transmission which can indicate the onset of epidemic outbreaks ${ }^{42}$. Current and future vector control programs should be assessed for their suitability of supplemental implementation of vector control measures to target regionally important diseases.

\section{Conclusion}

New vector control approaches are urgently needed to address rising resistances and changes in vector biting behavior. Their routine implementation is baffled not only by economic restraints but equally by a lack of uptake into international vector control guidelines, leaving countries hamstringed in implementing such programs. Larviciding programs often suffer from their alleged high costs and an allocation of funds almost solely towards adult vector control through bed nets. Additional approaches are needed to further curb intervention costs. Increasing the treatment intervals for larviciding, raising community involvement, and targeting several diseases at once could contribute to increased cost effectiveness of such interventions and facilitate their uptake into national and international control programs.

\section{Acknowledgements}

The author wants to thank the charitable Manfred Lautenschläger Foundation, Wiesloch, Germany for funding the EMIRA research project. We acknowledge financial support by Deutsche Forschungsgemeinschaft and Ruprecht-Karls-Universität Heidelberg within the funding program Open Access Publishing for publishing this article.

\section{Conflict of interest}

There are no conflicts of interest 


\section{References}

1. WHO | World malaria report 2017. WHO Available at: http://www. who.int/malaria/publications/world-malaria-report-2017/report/ en/. (Accessed: 6th February 2018)

2. Bhatt $\mathrm{S}$, Weiss DJ, Cameron E, et al. The effect of malaria control on Plasmodium falciparum in Africa between 2000 and 2015. Nature. 2015; 526: 207-211.

3. Macdonald G. The Epidemiology and Control of Malaria. (Oxford University Press, 1957).

4. WHO. Larval Source Management - A supplementary measure for malaria vector control. 2013.

5. Viana M, Hughes A, Matthiopoulos J, et al. Delayed mortality effects cut the malaria transmission potential of insecticide-resistant mosquitoes. Proc Natl Acad Sci USA. 2016; 113: 8975-8980.

6. Thomas MB, Read AF. The threat (or not) of insecticide resistance for malaria control. Proc Natl Acad Sci USA. 2016; 113: 8900-8902.

7. Strode C, Donegan S, Garner P, et al. The Impact of Pyrethroid Resistance on the Efficacy of Insecticide-Treated Bed Nets against African Anopheline Mosquitoes: Systematic Review and MetaAnalysis. PLOS Med. 2014; 11: e1001619.

8. Namountougou M, Simard F, Baldet T, et al. Multiple insecticide resistance in Anopheles gambiae s.l. populations from Burkina Faso, West Africa. PloS One. 2012; 7: e48412.

9. Fornadel CM, Norris LC, Glass GE, et al. Analysis of Anopheles arabiensis Blood Feeding Behavior in Southern Zambia during the Two Years after Introduction of Insecticide-Treated Bed Nets. Am J Trop Med Hyg. 2010; 83: 848-853.

10. Moiroux N, Gomez MB, Pennetier C, et al. Changes in Anopheles funestus biting behavior following universal coverage of long-lasting insecticidal nets in Benin. J Infect Dis. 2012; 206: 1622-1629.

11. Thomsen EK, Koimbu G, Pulford J, et al. Mosquito Behavior Change After Distribution of Bednets Results in Decreased Protection Against Malaria Exposure. J Infect Dis. 2017; 215: 790-797.

12. Becker N. Bacterial control of vector-mosquitoes and black flies. in Entomopathogenic bacteria: from laboratory to field application 383-398 (Springer, 2000).

13. Maheu-Giroux M, Castro MC. Impact of Community-Based Larviciding on the Prevalence of Malaria Infection in Dar es Salaam, Tanzania. PLOS ONE. 2013; 8: e71638.

14. Fillinger U, Lindsay SW. Suppression of exposure to malaria vectors by an order of magnitude using microbial larvicides in rural Kenya. Trop Med Int Health. 2006; 11: 1629-1642.

15. Fillinger U, Ndenga B, Githeko A, et al. Integrated malaria vector control with microbial larvicides and insecticide-treated nets in western Kenya: a controlled trial. BullWorld Health Organ. 2009; 87 655-665.

16. Geissbühler Y, Kannady K, Chaki PP, et al. Microbial Larvicide Application by a Large-Scale, Community-Based Program Reduces Malaria Infection Prevalence in Urban Dar Es Salaam, Tanzania. PLoS ONE. 2009; 4: e5107.

17. Majambere S, Lindsay SW, Green C, et al. Microbial larvicides for malaria control in The Gambia. Malar J. 2007; 6: 76.

18. Dambach P, Schleicher M, Stahl HC, et al. Routine implementation costs of larviciding with Bacillus thuringiensis israelensis against malaria vectors in a district in rural Burkina Faso. Malar J. 2016; 15: 380 .

19. Suaya JA, Shepard DS, Chang MS, et al. Cost-effectiveness of annual targeted larviciding campaigns in Cambodia against the dengue vector Aedes aegypti. Trop Med Int Health TM IH. 2007; 12: 1026-1036.
20. Maheu-Giroux M, Castro MC. Cost-effectiveness of larviciding for urban malaria control in Tanzania. Malar J. 2014; 13: 477.

21. The potential impact of integrated malaria transmission control on entomologic inoculation rate in highly endemic areas. - PubMed - NCBI. Available at: https://www.ncbi.nlm.nih.gov/bmed/?ter$\mathrm{m}=\mathrm{The}+$ potential+impact+of+integrated+malaria+transmission+control+on+entomologic+inoculation+rate+in+highly+endemic+areas. (Accessed: 11th January 2018)

22. Dambach P, Traoré I, Becker N, et al. EMIRA: Ecologic Malaria Reduction for Africa - innovative tools for integrated malaria control. Glob Health Action. 2014; 7.

23. Dambach P. Reduction of malaria vector mosquitoes in a large scale intervention trial in rural Burkina Faso using Bti based larval source management. Manuscript in preparation. 2017.

24. Dambach P, Machault V, Lacaux JP, et al. Utilization of combined remote sensing techniques to detect environmental variables influencing malaria vector densities in rural West Africa. Int J Health Geogr. 2012; 11: 8 .

25. Dambach P, Sié A, Lacaux JP, et al. Using high spatial resolution remote sensing for risk mapping of malaria occurrence in the Nouna district, Burkina Faso. Glob Health Action. 2009; 2.

26. Machault V, Vignolles C, Pagès F, et al. Risk Mapping of Anopheles gambiae s.l. Densities Using Remotely-Sensed Environmental and Meteorological Data in an Urban Area: Dakar, Senegal. PLoS ONE. $2012 ; 7$.

27. Dambach P, Louis VR, Kaiser A, et al. Efficacy of Bacillus thuringiensis var. israelensis against malaria mosquitoes in northwestern Burkina Faso. Parasit. Vectors. 2014; 7: 371.

28. Fillinger U, Knols BGJ, Becker N. Efficacy and efficiency of new Bacillus thuringiensis var. israelensis and Bacillus sphaericus formulations against Afrotropical anophelines in Western Kenya. Trop Med Int Health. 2003; 8: 37-47.

29. Diédhiou SM, Konaté L, Doucouré S, et al. [Effectiveness of three biological larvicides and of an insect growth regulator against Anopheles arabiensis in Senegal]. Bull Soc Pathol Exot. 1990. 2017; 110: $102-115$

30. Mbare 0, Lindsay SW, Fillinger U. Dose-response tests and semi-field evaluation of lethal and sub-lethal effects of slow release pyriproxyfen granules (Sumilarv®0.5G) for the control of the malaria vectors Anopheles gambiae sensu lato. Malar J. 2013; 12: 94.

31. Abad-Franch F, Zamora-Perea E Luz SLB. Mosquito-Disseminated Insecticide for Citywide Vector Control and Its Potential to Block Arbovirus Epidemics: Entomological Observations and Modeling Results from Amazonian Brazil. PLoS Med. 2017; 14: e1002213.

32. Caputo B, Ienco A, Cianci D, et al. The 'auto-dissemination' approach: a novel concept to fight Aedes albopictus in urban areas. PLoS Negl Trop Dis. 2012; 6, e1793.

33. Lwetoijera D, Harris C, Kiware S, et al. Effective autodissemination of pyriproxyfen to breeding sites by the exophilic malaria vector Anopheles arabiensis in semi-field settings in Tanzania. Malar J. 2014; 13: 161.

34. Kiware SS, Corliss G, Merrill S, et al. Predicting Scenarios for Successful Autodissemination of Pyriproxyfen by Malaria Vectors from Their Resting Sites to Aquatic Habitats; Description and Simulation Analysis of a Field-Parameterizable Model. PLoS ONE. 2015; 10.

35. Dambach P. Community perception and acceptance of environmental larviciding against malaria with Bti formulations in Burkina Faso. Manuscript in preparation. 2017.

36. Becker N. Community participation in the operational use of microbial control agents in mosquito control programs. Bull Soc Vector Ecol. 1992; 17: 114-118. 
37. da Silva NS, da Silva-Nunes M, Malafronte RS, et al. Epidemiology and control of frontier malaria in Brazil: lessons from community-based studies in rural Amazonia. Trans R Soc Trop Med Hyg. 2010; 104: 343-350.

38. Chaki PP, Govella NJ, Shoo B, et al. Achieving high coverage of larvalstage mosquito surveillance: challenges for a community-based mosquito control programme in urban Dar es Salaam, Tanzania. Malar J. 2009; 8: 311 (2009).

39. Waggoner J. Brichard J, Mutuku F, et al. Malaria and Chikungunya Detected Using Molecular Diagnostics Among Febrile Kenyan Children. Open Forum Infect Dis. 2017; 4: ofx110.
40. Ward T, Samuel M, Maoz D, et al. Dengue data and surveillance in Tanzania: a systematic literature review. Trop Med Int Health TM IH. 2017; 22: 960-970.

41. Horstick O, Runge-Ranzinger S. Protection of the house against Chagas disease, dengue, leishmaniasis, and lymphatic filariasis: a systematic review. Lancet Infect Dis. 2017.

42. Louis VR, Phalkey R, Horstick O, et al. Modeling tools for dengue risk mapping - a systematic review. Int J Health Geogr. 2014; 13: 50. 